\title{
Collaborative Map Matching in Nav2Nav
}

\author{
Monsak Socharoentum and Hassan A. Karimi \\ Geoinformatics Laboratory, School of Information Sciences \\ University of Pittsburgh135 N. Bellefield, Pittsburgh, PA, 15260, US. \\ monsak.s@gmail.com; hkarimi@sis.pitt.edu
}

\begin{abstract}
Although in-car navigation systems are becoming commonplace, cars travelling in urban canyon areas still suffer from poor positional accuracy and limited visibility of GPS satellites due to signal blockage. As a result, map matching accuracy and navigation performance may not reach the required level for many applications. Researchers have tried to address positional inaccuracy in navigation systems using various techniques, such as map matching, augmentation, and differential GPS. However, some void still exist as these techniques require intensive computation, static base stations, or installation of extra equipment. In this paper, we propose Collaborative Map Matching (CMM) which is a novel technique aiming to improve map matching accuracy in Nav2Nav. CMM is based on differential GPS, high quality road map, and collaborative computation. CMM does not require intensive computation, static base stations, or extra equipment installed in cars. The main requirement is for cars to work collaboratively, through Nav2Nav, to help one another. Simulation of CMM shows that improved GPS positional accuracy obtained by one car can be shared to improve the map matching accuracy of other nearby cars.
\end{abstract}

Index Terms - DGPS, Map Matching, Car Navigation, Nav2Nav

\section{INTRODUCTION}

It is known that in-car navigation systems suffer from limited visibility of GPS satellites in urban canyons due to signal blockage and multipath. These difficulties generally result in poor GPS positional accuracy. A field test in London revealed that GPS positions may be more than 50 meters away from the true position [3]. Another study in Hong Kong found even larger positional errors, sometimes larger than 80 meters [2]. The effect of these large positional errors manifests as low map matching accuracy which directly affects navigation performance.

Map matching is a process of locating a vehicle position on a road segment [9]. First, a position must be calculated using positioning sensors (such as GPS or GPS integrated with dead reckoning), and then the calculated position is input to the map matching algorithm. Map matching composes of two major steps: (1) locating the correct road segment on which the vehicle is travelling, and (2) projecting the input position onto the road segment. The projected position is the data that is used for navigation and tracking. Map matching is an essential component of a navigation system whose performance heavily depends on the quality of the road map database [25].

Navigation-to-Navigation (Nav2Nav) is a peer-to-peer paradigm where navigation systems in nearby cars can communicate with each other to address specific problems such as crash avoidance [23]. Nav2Nav is different than V2V and V2I in that in Nav2Nav a road database is used as a model of the navigation environment and navigation and tracking information, such as routes, in addition to raw position data, can be exchanged between cars. This paper presents a novel technique called Collaborative Map Matching (CMM) aiming to improve map matching accuracy in Nav2Nav. CMM is a collaborative computing environment using Differential GPS (DGPS) method and high quality road maps. DGPS is defined [5] as "a method to improve positioning or timing performance of GPS using one or more base stations at known locations, each equipped with at least one GPS receiver.” DGPS requires base station(s) to provide corrections to the raw pseudorange measured by rover(s). By calculating rover's position using the provided corrections, the rover could achieve better positional accuracy.

In urban canyons, a high quality road map usually has higher positional accuracy than GPS positions. For instance, Topologically Integrated Encoding and Referencing (TIGER; version 2009) road map has positional accuracy better than 10 meters in urban areas [6]. Another example is NAVSTREETS $®$ whose road map data have absolute positional accuracy as good as \pm 5 meters and relative positional accuracy as high as \pm 1 meter for some segments [7].

CMM is built on collaboration between cars with good GPS positional accuracy and cars with poor positional accuracy. The contribution of CMM is that it improves map matching accuracy in Nav2Nav, leading to enhanced navigation and tracking performances in navigation systems.

The paper is structured as follows. Section II provides a background to DGPS and pseudorange correction. Section III discusses CMM in detail. Section IV discusses the experiments and results. Finally, discussions, conclusions, and future research are provided in Section V.

\section{BACKGROUND}

DGPS is aimed to improve GPS positional accuracy from meters level to centimeters level. Example DGPS implementation include experiments with network-based 
Real-Time Kinematic (RTK) GPS [15, 16, 17, 18, and 19] in Japan, Singapore, Germany, and Austria. Network-based RTK GPS requires GPS base stations established permanently and linked together to form a network that covers the operation area. GPS receivers which have accessibility to the broadcasted error correction can then exploit it to enhance their positional accuracy.

Wide-Area DGPS (WADGPS) aims to attain meter-level accuracy over a large coverage region (e.g., country or continent). WADGPS requires a network of base stations distributed over a region similar to network-based RTK GPS. Examples of WADGPS are the Wide Area Augmentation System (WAAS) [20, 11] in the U.S., the European Geostationary Navigation Overlay Service (EGNOS) [21] in Europe, and the Multi-functional Satellite Augmentation System (MSAS) [21] in Japan. These three systems are all categorized as satellite-based augmentation systems which provide error correction to the users in the service region through a separate group of satellites. WAAS, EGNOS, and MSAS were initially designed for aircraft navigation but later EGNOS specifically extended its service (called SISNet [22]) to include land-based navigation applications. The data link between SISNet and its users spans over the Internet and/or a cellular network.

CMM is different from network-based RTK-GPS and WADGPS in that CMM does not require a permanent network of base stations. In addition, given that CMM is for Nav2Nav, which is a peer-to-peer collaboration among navigation systems in cars, there is no need for a third party service. In addition, compared to [24] which needs complicated statistical models for map matching, CMM does not require high computing resources, and augmentation systems installed.

DGPS is a well-known method to obtain better positional accuracy of a rover using one or more base station(s). Each base station must have known coordinates which are accurately surveyed prior to DGPS operation. The coordinates should be more accurate (e.g., at centimeter-level) than general standalone GPS positional accuracy (e.g., 5-20 m.).

DGPS techniques can be grouped into three categories [5]: positioning-based (absolute or relative differential positioning); area-based (local, regional, or wide); and RFbased (code or carrier). The remainder of this section is devoted to the discussion of Pseudorange Correction (PRC) because it is utilized in CMM.

Fig. 1 shows the DGPS concept where one base station with known coordinates $(\mathrm{X}, \mathrm{Y}, \mathrm{Z})_{\mathrm{S}}$ and one rover are linked through a communication link. Rover's GPS coordinates are denoted by $(\mathrm{X}, \mathrm{Y}, \mathrm{Z})_{\mathrm{GPS}}$. PR1, PR2, and PR3 denote Pseudoranges (PR) observed by the base station (PRs are the distance between the GPS receiver and visible satellites). PR4 and PR5 denote Pseudoranges observed by the rover. The vector between the base station and the rover is called the Baseline. In Fig. 1, only satellites \#2 and \#3 are locked by both the base station and rover, therefore, only the error correction for the two satellites can be used by the rover.

To obtain 3D coordinates, a GPS receiver has to observe at

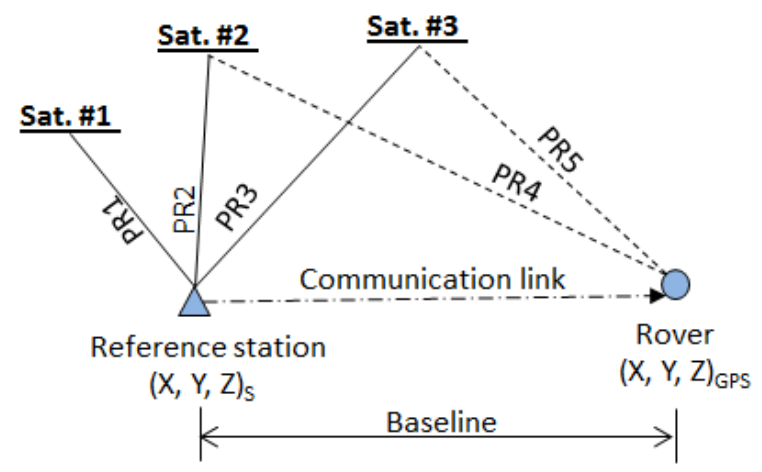

Fig. 1. Differential GPS.

least four satellites and the signal travel time from each satellite. In DGPS, the process is performed inversely, that is the known coordinates of the base station and satellites will be used to calculate the ranges spanning from the station to each satellite. Ranges (R) are more accurate than PR as they are derived from a geometrical equation instead of observation which is usually impacted by several sources of errors (e.g., atmospheric delay, satellite clock error). PRC is the difference between $\mathrm{R}$ and $\mathrm{PR}$, and $\mathrm{R}$ is always shorter than PR.

Generally, GPS error sources are highly correlated over space and time. DGPS exploits these correlations by applying PRC from base station(s) to rover(s). Errors in base station's PR for visible satellites are expected to be very similar to those experienced by nearby rovers.

\section{COLlaborative MAP MATChING (CMM)}

In this paper, "Station" refers to base station and "Rover" to rover. An in-car navigation system travelling on a road network and capable of Nav2Nav could be either Station or Rover; Station is a navigation system experiencing high positional accuracy and Rover is a navigation system experiencing poor positional accuracy at a given location and time. To improve Rover's map matching accuracy, three assumptions are made in CMM: (1) the GPS positional accuracy varies over space and time, (2) the high quality road map contains higher positional accuracy than standalone GPS positions, and (3) a communication channel (either one-way or two-way) is available to both Rover and Station.

An example of the first assumption is a car in an urban canyon which may experience poor positional accuracy (Rover), due to signal blockage, while another nearby car under open sky may experience high GPS positional accuracy (Station). The range of positional accuracy in a high quality road map may be 5-10 meters ([6] and [7]) compared to the range of standalone GPS positions observed in urban canyon which may be 50-80 meters ([2] and [3]). The last assumption states that all cars are capable of Nav2Nav. The CMM algorithms were designed to work under both one-way and two-way communication. Note that communication range is not of a concern in CMM. The main difference is that with long-range communication a larger number of Stations nearby a Rover may be possible compared to number of Stations with 
short-range communication. In case of several candidate Stations, CMM can select the one with the highest possibility of improving Rover's map matching accuracy.

\section{A. DGPS and CMM}

CMM is partly based on a DGPS technique. DGPS can improve Rover's GPS positional accuracy with the criterion that Station and Rover have to be under similar atmospheric effects. Based on these common effects, the Station could calculate the PRC using coordinates with higher accuracy and shares them with the Rover. This is because the Station and the Rover in close proximity experience similar atmospheric effects and less difference in delays. The atmospheric similarity requirement is suitable for urban/city road networks since usually many cars in close proximity travel on them.

DGPS assumes that at least one GPS receiver (base station) is fixed at a highly accurate surveyed position (e.g., centimeters level accuracy). In CMM, where cars can assume the role of Stations, there are no fixed stations. As a result, highly accurate surveyed positions for CMM are impossible to achieve. In CMM, a map matched Station's GPS position using a high quality road map is considered as the highest accuracy possible for that position (similar to surveyed position of base station in DGPS). Nevertheless, navigation systems equipped with high quality road map and Stations with high GPS positional accuracy are expected to improve GPS positional accuracy at Rovers.

\section{B. Nav2Nav's Peer-to-Peer Architecture}

In CMM a peer is any car equipped with in-car navigation system capable of Nav2Nav. A peer could either assume the role of Station or Rover at any time depending on the environment. For example, a peer in an open sky environment would more often be designated as a Station, while a peer in an urban canyon is more likely to be designated as a Rover. CMM designates a peer with poor positional accuracy as Rover and finds PRCs from other peers which are designated as Stations. As a Rover moves to a new environment with high positional accuracy, its designation may change to Station.

The peer-to-peer architecture in Nav2Nav implies that: (1) peers can directly exchange information with one another and (2) computation is performed locally and independent of other peers. The CMM algorithms presented and discussed in this paper take into account these two characteristics of Nav2Nav.

\section{CMM Algorithms}

CMM algorithms were designed based on two types of communication: one for one-way communication, and one for two-way communication.

For one-way communication, CMM assumes that Station and Rover can communicate only unidirectionally. In this case, a peer acting as Station must continuously broadcast its PRCs to Rovers within the communication range. The Rover receives the broadcasted PRCs, evaluates their quality, filters them if needed, and applies the correction.

Fig. 2 (a) shows Phase 1 of the algorithm (performed by Station). The Station starts with S1: calculate GPS position and Horizontal Dilution of Position (HDOP), then S2: map match the GPS position, S3: calculate PRC for visible GPS satellites, and finally, S4: broadcast PRCs and HDOP. Note that Stations that have high HDOP (e.g. > 4; the minimum theoretical HDOP is 1) would stop at S1 as a high HDOP usually indicates low positional accuracy.

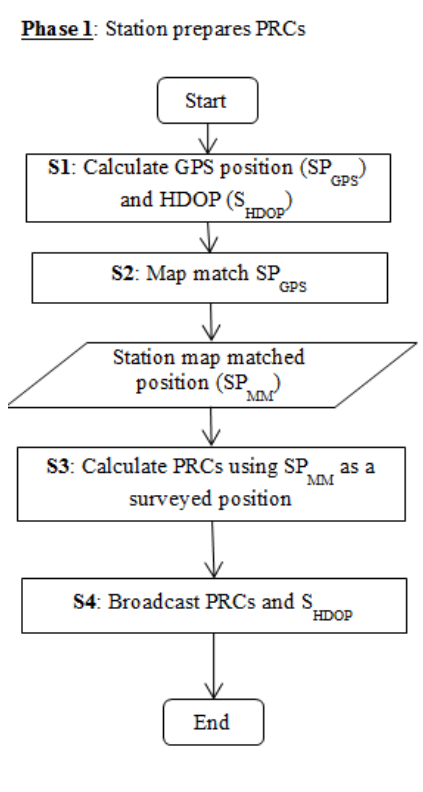

(a)

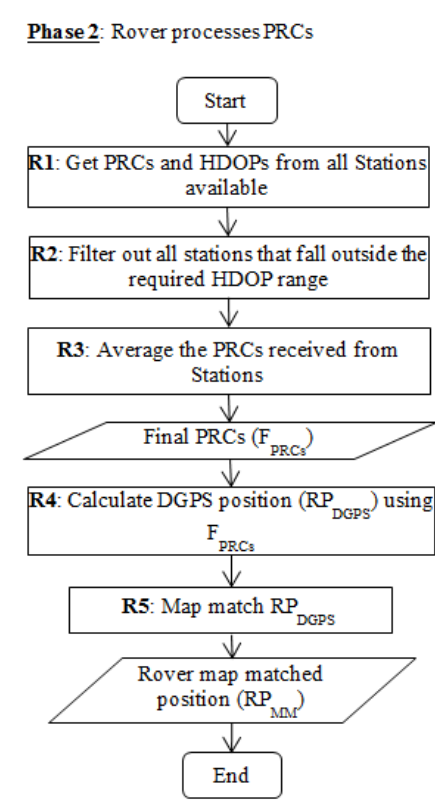

(b)
Fig. 2. CMM algorithm for one-way communication.

Fig. 2 (b) shows Phase 2 of the algorithm (performed by Rover). The Rover starts with R1: retrieve PRCs and HDOPs from all available Stations, then R2: filter out Stations with high HDOP values, R3: average the PRC for each visible GPS satellite, R4: calculate DGPS position using the average PRCs, and finally, R5: map match the DGPS position. Note that in one-way communication, each Station must continually calculate PRCs and HDOP and broadcast them to Rovers.

For two-way communication, CMM assumes that Station and Rover can communicate bidirectionally. Overall, two-way communication requires less computation and communication, compared to one-way communication, since a Station can wait until a Rover sends a request. The algorithm is composed of three phases as shown in Fig. 3.

Fig. 3 (a) shows Phase 1 of the algorithm (performed by Rover). The Rover starts from R1: calculate its GPS position and HDOP, then R2: define expected HDOP range (EHD), R3: send Rover's GPS position and EHD to all Stations within the communication range, and R4: wait for response(s) from Station(s).

Fig. 3 (b) shows Phase 2 of the algorithm (performed by Station). When a Station receives a request from a Rover, the Station starts at S1: read $\mathrm{RP}_{\mathrm{GPS}}$ and EHD received and save them in the memory, then S2: calculate Station's GPS position and its HDOP $\left(\mathrm{S}_{\mathrm{HDOP}}\right)$, S3: check if $\mathrm{S}_{\mathrm{HDOP}}$ falls within EHD (if it does, $\mathrm{S} 4$, if not, end the process), $\mathrm{S} 4$ : map match $\mathrm{SP}_{\mathrm{GPS}}$ and get map matched position ( $\left.\mathrm{SP}_{\mathrm{MM}}\right)$, S5: calculate PRCs using $\mathrm{SP}_{\mathrm{MM}}$, and finally, S6: send PRCs back to Rover. 
Fig. 3 (c) shows Phase 3 of the algorithm (performed by Rover). When Rover receives PRCs from all potential Stations (responding back with a timeout period), it resumes to R5: read all PRCs from all potential Stations, R6: average the PRCs for each satellite observable by the Rover (the reason to average the PRCs is explained in Section V.A and confirmed by the empirical result discussed in Section IV.C), R7: calculate DGPS position ( $\mathrm{RP}_{\text {DGPS }}$ ), and finally, R8: map match $\mathrm{RP}_{\mathrm{DGPS}}$ for Rover (map matched position is at $\mathrm{RP}_{\mathrm{MM}}$ ). satellites observed to be four (minimum satellites required for calculating 3D position and HDOP); (c) number of Stations varied between 1,5 , and 20. The Stations were located in close proximity to the Rover (the maximum distance between Station and Rover is around $2 \mathrm{~km}$ and all Stations are located within $10 \mathrm{~km}^{2}$ ).

The data used in the experiments are as follows. GPS Almanac file from U.S. Coast Guard Navigation Center [10] for May 15, 2011, was used to simulate GPS satellites orbits and orientations. NAVSTREETS ${ }^{\circledR}$ (a road map data set

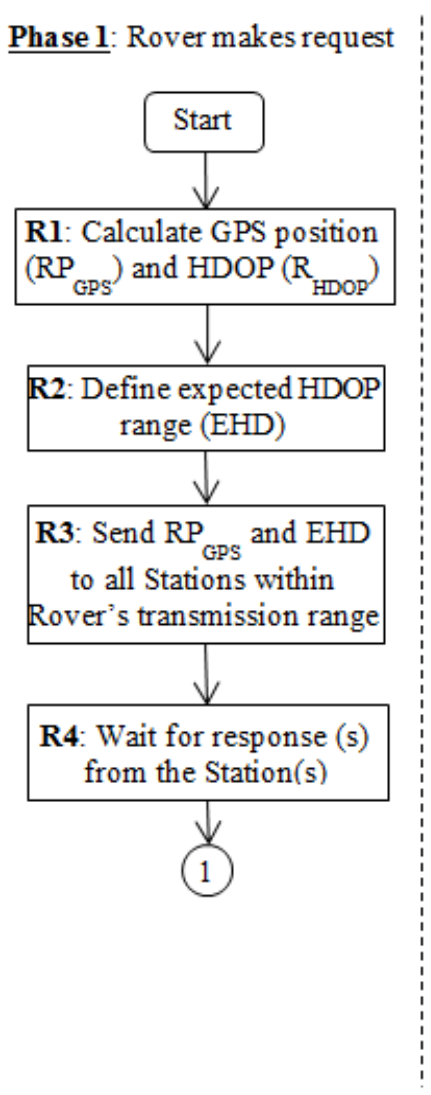

(a)

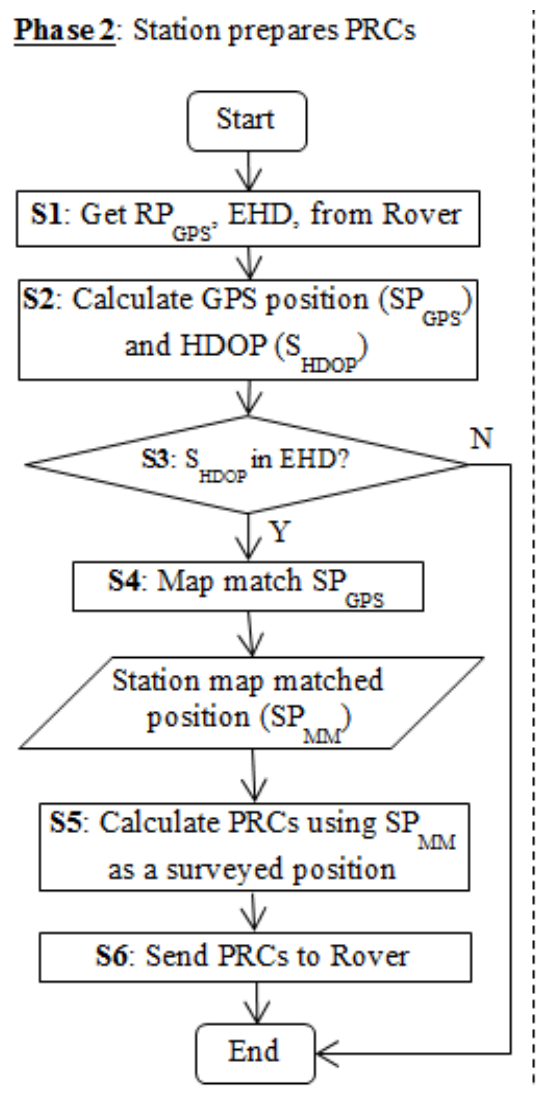

(b)

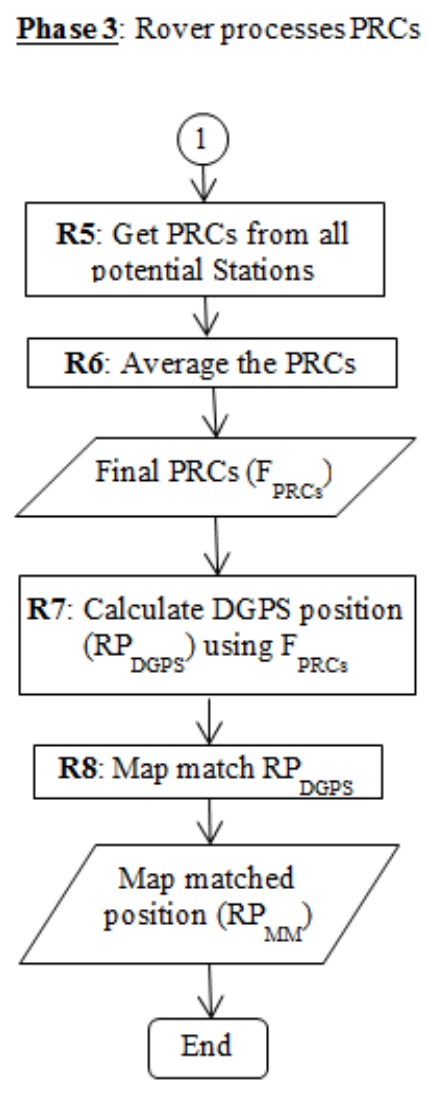

(c)

Fig. 3. CMM algorithm for two-way communication.

\section{SimUlATION AND RESULTS}

\section{A. Experiment}

Experiments were simulated to address three research questions as follows. RQ1: Can CMM produce better accuracy than DGPS? RQ2: What is the required number of Stations to give the best map matching accuracy for a Rover? RQ3: What is the relationship between the Rover HDOP and the map matching accuracy?

The following experiment settings were considered: (a) all Stations under open sky. This means no signal blockage in the environment. All GPS satellites with elevation greater than 2 degrees are accepted; (b) only one peer designated as Rover. To simulate the Rover experiencing poor GPS positional accuracy, its visibility was masked to limit the number of provided by NAVTEQ $\left.{ }^{\circledR}[7]\right)$ was used as road database for all Stations and Rovers. The road network represented the city of Pittsburgh, PA, USA. A high-resolution aerial image from PASDA [8] was used as background for manual digitization of travelling path of the Stations and Rover. Twenty one seed positions (20 Stations and 1 Rover) were extracted from the travelling path. These seed positions were fed to the Constellation Toolbox (the simulation software) to retrieve simulated GPS positions.

\section{B. Simulation}

The simulation was implemented using a MATLAB-based GPS simulation software called Constellation Toolbox, version 8.0 [12]. The software was run under MATLAB environment version 7.12. We used the functions provided by Constellation Toolbox to acquire information for the simulation; namely, satellite orientation, GPS position, 
HDOP, pseudorange, pseudorange correction, atmospheric model, and DGPS position. We imported the city of Pittsburgh's road network from NAVSTREET ${ }^{\circledR}$ into PostGIS version 1.5.2 [13] and PostgreSQL version 9.0 [14]. PostGIS provides two geometrical functions which are used for map matching: a function for finding the nearest road segment for a point, and a function for projecting the point onto the segment. To evaluate positional accuracy, we used a point-topoint distance function provided by PostGIS. All distances were projected from a geographic coordinate system into a Cartesian coordinate system using meters as the distance unit.

Four scenarios were simulated (see Table I). Scenarios A and B were designed to answer RQ1 and Scenarios B, C and $\mathrm{D}$ were designed to answer RQ2.

TABLE I

SCENARIOS IMPLEMENTED IN THE SIMULATION

\begin{tabular}{|c|r|r|c|}
\hline Scenarios & \# of Station & \# of Rover & $\begin{array}{c}\text { CMM/plain DGPS } \\
\text { technique applied }\end{array}$ \\
\hline A & 20 & 1 & Plain DGPS \\
\hline B & 20 & 1 & CMM \\
\hline C & 1 & 1 & CMM \\
\hline D & 5 & 1 & CMM \\
\hline
\end{tabular}

Table II shows number of visible satellites, HDOP, and GPS positional accuracy for Stations and Rover calculated at 12:00 am on May 15, 2011. The first row summarizes information for the 20 Stations used in the simulation. No mask was applied to any Stations; this was to simulate open sky. The second row is for the Rover, where 210 combinations generated by selecting all possible sets of 4 satellites out of the 10 visible satellites. Masks were generated and applied to filter out the unwanted satellites; this was to simulate urban canyon. Note that no multipath effect was taken into account because the focus of this work is satellite blockage.

As shown in Table II, HDOP of the 20 Stations varies slightly (1.64 - 1.66) as the Stations are located close to one another and have the same 10 visible satellites. The Stations' average HDOP (1.65) is far better than that of Rover's $(1,206)$. GPS positional accuracy range for the 20 Stations is very narrow (min: $5.73 \mathrm{~m}$, max: $5.83 \mathrm{~m}$, and avg: $5.81 \mathrm{~m}$ ), however, it is better than the range of Rover's positional accuracy (min: $0.42 \mathrm{~m}$, max: 42,824 m, and avg: $465.6 \mathrm{~m}$ ).

TABLE II

INFORMATION ON STATIONS AND ROVER

\begin{tabular}{|l|c|c|c|c|c|c|c|}
\hline \multirow{2}{*}{ CMM Roles } & $\begin{array}{c}\text { \# of } \\
\text { satellites } \\
\text { seen }\end{array}$ & Min & Max & Avg & Min & Max & Avg \\
\hline 20 Stations & 10 & 1.64 & 1.66 & 1.65 & 5.73 & 5.83 & 5.81 \\
\hline Rover (210 cases) & 4 & 1.18 & 171,754 & 1,206 & 0.42 & 42,824 & 465.6 \\
\hline
\end{tabular}

\section{Results}

An analysis of the empirical results (see Table III) indicates that CMM can significantly improve map matching accuracy (78\%-87\%). CMM has better accuracy compared to DGPS $(7.7 \%$ - 19.6\%). The improvements are evident in all simulated Rover HDOP ranges.
TABLE III

ROVER MAP MATCHED POSITIONAL ERROR

\begin{tabular}{|c|c|c|c|c|c|c|}
\hline \multirow{3}{*}{$\begin{array}{c}\text { Rover } \\
\text { HDOP } \\
\text { ranges } \\
(x=1.645)\end{array}$} & \multicolumn{6}{|c|}{ Average map match positioning error (meter)* } \\
\hline & \multirow{2}{*}{$\begin{array}{c}\text { No } \\
\text { correction } \\
\text { (A) }\end{array}$} & \multirow{2}{*}{$\begin{array}{c}\text { With } \\
\text { CMM (B) }\end{array}$} & \multirow{2}{*}{$\begin{array}{c}\text { Simple } \\
\text { DGPS } \\
\text { (C) }\end{array}$} & \multicolumn{3}{|c|}{ \% Difference } \\
\hline & & & & (A)-(B) & $(\mathrm{A})-(\mathrm{C})$ & (C)-(B) \\
\hline $2 x$ to $4 x$ & 8.88 & 1.89 & 2.35 & 78.7 & 73.5 & 19.6 \\
\hline $2 \mathrm{x}$ to $6 \mathrm{x}$ & 18.42 & 2.40 & 2.87 & 87.0 & 84.4 & 16.4 \\
\hline $2 \mathrm{x}$ to $8 \mathrm{x}$ & 17.80 & 2.38 & 2.81 & 86.6 & 84.2 & 15.3 \\
\hline $2 \mathrm{x}$ to $10 \mathrm{x}$ & 20.11 & 2.67 & 3.12 & 86.7 & 84.5 & 14.4 \\
\hline $2 \mathrm{x}$ to $20 \mathrm{x}$ & 28.12 & 4.26 & 4.82 & 84.9 & 82.9 & 11.6 \\
\hline $2 \mathrm{x}$ to $30 \mathrm{x}$ & 35.89 & 5.20 & 5.70 & 85.5 & 84.1 & 8.8 \\
\hline $2 x$ to $50 x$ & 39.08 & 5.98 & 6.48 & 84.7 & 83.4 & 7.7 \\
\hline
\end{tabular}

* compared to the map match positions of the seed positions

TABLE IV

DISTRIBUTION OF HDOP RANGES

\begin{tabular}{|c|r|r|}
\hline HDOP ranges & Distribution of cases & Percentage \\
\hline$>50 \mathrm{x}(>82.25)$ & 28 & 13.3 \\
\hline$<2 \mathrm{x}(<3.29)$ & 62 & 29.5 \\
\hline $2 \mathrm{x}$ to $50 \mathrm{x}$ & 120 & 57.1 \\
\hline Sum & 210 & 100.0 \\
\hline
\end{tabular}

Of the 210 cases, the HDOP range (2x to $50 x$ ) covers $57.1 \%$ as shown in Table IV. This implies that the improvement shown in Table III could be experienced $\sim 57 \%$ of the time. If we do not count the situations when a Rover has relatively good HDOP $(<2 \mathrm{x})$, then 62 cases (Table IV, second row) can be removed from the analysis. Using the remaining 148 cases, the percentage of HDOP range $2 \mathrm{x}$-to-50x is very high (81\%) which is calculated as follows $100 *[120 /(210-62)]$. That is to say, when Rover experiences poor HDOP, the improvement shown in Table III could be experienced $\sim 81 \%$ of the time.

To analyze the impact of the number of Stations used, the
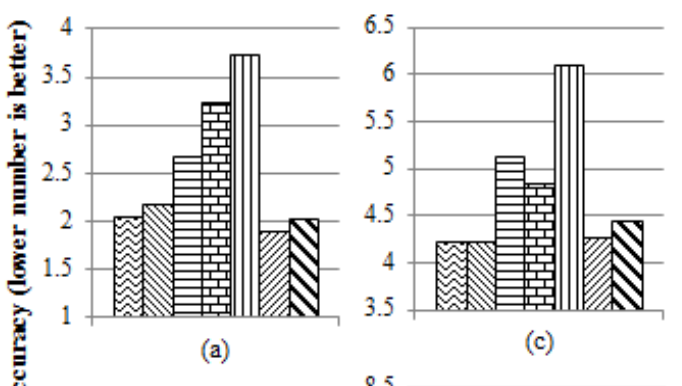

图

⿶S_ $\# 18$

日S_\#2

圆 $\mathrm{S}_{-} \neq 20$

m S_ $\# 19$

v20_stations

(c)

Q5_stations

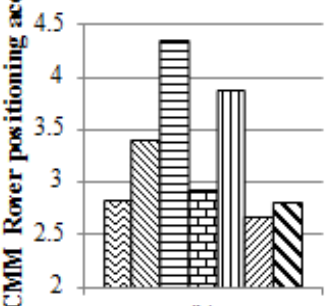

(b)

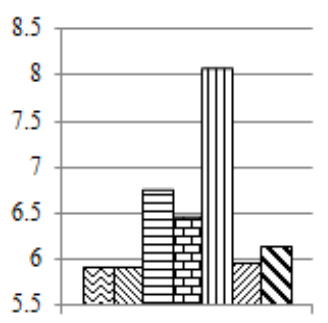

(d)
Fig. 4. Relation between number of Stations and accuracy.

variations of map matching accuracy for Scenarios B, C, and D (see Table I) are plotted in Fig. 4. Fig. 4 (a), (b), (c), and (d) show plots for four HDOP ranges (i.e. $2 x-4 x, 2 x-10 x, 2 x-20 x$, and $2 \mathrm{x}-50 \mathrm{x})$, respectively. The differences between using 1,5 
and 20 Stations are shown in bar graph form. The first five bars (from left) in Fig. 4 (a), (b), (c), and (d) represent the result from using five different Stations individually (Station ID \#1, \#18, \#2, \#20, and \#19). The sixth and seventh bars show the use of average PRCs from 20 and 5 Stations, respectively.

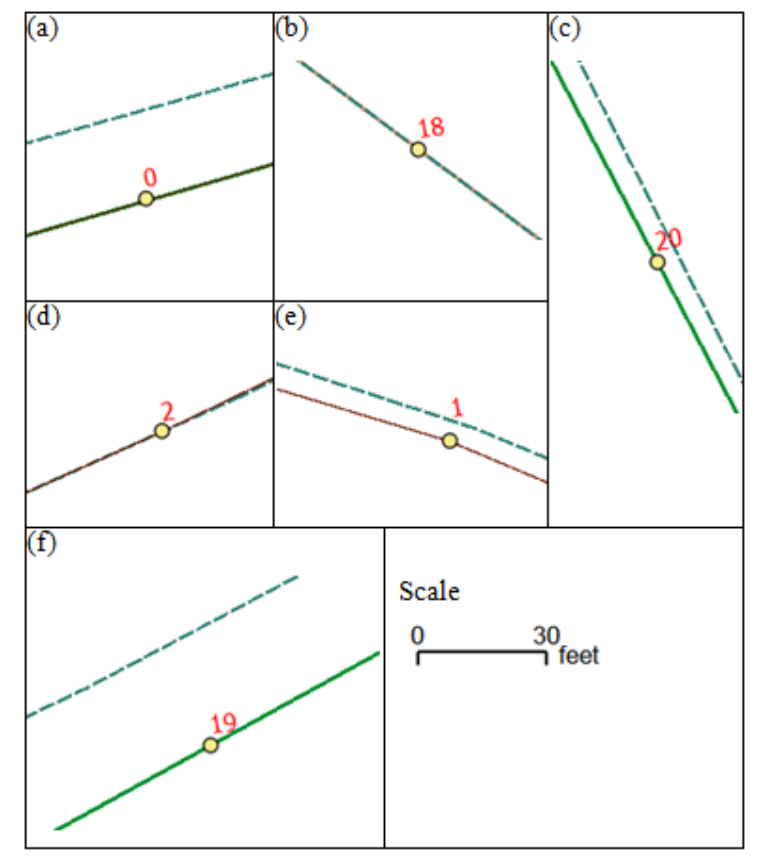

Fig. 5. Orientation of Rover (\#0) and five Stations (\#1, \#2, \#18, \#19, and \#20).

The average of PRCs using 20 Stations can deliver the best accuracy, or at least close to the best acquired from individual Stations (Station ID \#1 and \#18), compared to the average of PRCs using 5 Stations. We selected the 5 individual Stations (Station ID\#1, \#18, \#2, \#20, and \#19) based on their positional orientations relative to the corresponding road segments. Fig. 5(a) shows the orientation of the Rover and Fig. 5(b) to (f) show the 5 Stations. The dotted line represents a road segment from NAVSTREET ${ }^{\circledR}$ which is a proxy for a road database installed in a navigation system. The solid line represents the real travel paths. The deviation of Rover position, see Fig. 5 (a), relative to the corresponding road segment is in south-east direction. Station ID \#18, \#20 and \#1 were chosen, see Fig. 5 (b), (c), and (f), because they deviated in south-west direction (opposite to Rover's orientation). Station ID \#2 and \#19 were chosen because they deviated in south-east direction (similar to Rover's orientation). These two groups are the two extreme cases in terms of positional orientation.

\section{DisCUSSION, CONCLUSION, AND FUTURE RESEARCH}

\section{A. Discussion}

A major characteristic of CMM in Nav2Nav is that it aims to determine optimal PRCs compared to simply using an uncorrected GPS position. CMM performs five steps to find an optimal PRC: (1) select a set of Stations in open sky, (2) retrieve GPS positions of those Stations, (3) map match the positions onto associated road segments, (4) calculate PRCs using the map matched positions, and (5) average the PRCs.

The question is: How would map matching help improve PRCs quality?

Generally, Stations calculate PRCs based on a highly accurate known position. A PRC is then used to correct the raw pseudorange measurement. It is obvious that with higher positional accuracy of a known position, a more accurate PRC would be achieved. More accurate PRCs would lead to improved accuracy when applied by the Rover.

To address the question above, GPS positional errors relative to road segments and map matched positions need to be analyzed. We use map matched position for a base line because the goal of CMM is to improve map matching accuracy.

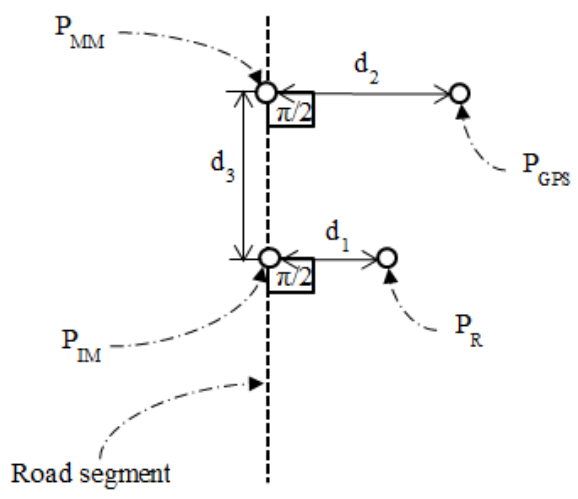

Fig. 6. Notations of spatial elements.

Let $\mathrm{P}_{\mathrm{GPS}}$ denote GPS position (estimation of the real position denoted by $\mathrm{P}_{\mathrm{R}}$ ), $\mathrm{P}_{\mathrm{IM}}$ denote map matched position of $\mathrm{P}_{\mathrm{R}}$, and $\mathrm{P}_{\mathrm{MM}}$ denote map matched position of $\mathrm{P}_{\mathrm{GPS}}$ (see Fig. 6). The error distance is classified into two components which are error distance across road segment denoted by $\mathrm{d}_{2}$ and along road segment denoted by $d_{3}$. The projected distance of $P_{R}$ is denoted by $\mathrm{d}_{1}$. The dotted line represents the associated road segment.

Map matching eliminates error distance across a road segment $\left(\mathrm{d}_{2}\right)$. With a high quality road map, if GPS positional accuracy is not large (e.g., positional error $<40 \mathrm{~m}$ ), the likelihood of finding the correct road segment is high. After the second step (point projection), $\mathrm{d}_{2}$ will be eliminated. Therefore, when acquiring $\mathrm{P}_{\mathrm{MM}}$ (map matching $\mathrm{P}_{\mathrm{GPS}}$ ), $\mathrm{d}_{2}$ will be removed, and this makes $\mathrm{P}_{\mathrm{MM}}$ more accurate than $\mathrm{P}_{\mathrm{GPS}}$.

CMM reduces error distance along a road segment $\left(\mathrm{d}_{3}\right)$. Even though $d_{2}$ could be eliminated via map matching, $d_{3}$ still exists as part of $\mathrm{P}_{\mathrm{MM}}$. However, when a large number of Stations is used, influence of $d_{3}$ could be reduced.

Considering a number of GPS positions measured independently in Nav2Nav, their positional error would propagate in all directions evenly. When normalizing the errors onto two directions (e.g., along and across road segment), the error along road segment $\left(\mathrm{d}_{3}\right)$ should propagate in both directions (toward and backward) relative to $\mathrm{P}_{\mathrm{IM}}$. Consequently, the vector sum of $d_{3}$ of all Stations should be 
close to zero in size. Accordingly, averaged PRCs calculated based on different GPS map matched positions should have less (or no) effect from $\mathrm{d}_{3}$ due to the cancellation in directions.

Finally, CMM should minimize the effect of two projected directional errors $\left(d_{2}\right.$ and $\left.d_{3}\right)$. In contrast, $d_{2}$ and $d_{3}$ would not be eliminated if only the standalone GPS position ( $\left.\mathrm{P}_{\mathrm{GPS}}\right)$ is used to calculate PRCs.

\section{B. Conclusions}

Compared to standalone GPS map matching, it was shown, through simulation and experiments, that CMM can help a Rover improve its map matching accuracy by $78 \%$ to $87 \%$. It was also shown that compared to DGPS, CMM can help a Rover improve its map matching accuracy by $7 \%$ to $20 \%$. CMM is superior over current techniques (e.g., WAAS, EGNOS, SISNet, network-based RTK or DGPS) in several ways. First, CMM does not require installation and operation of permanent base stations to track GPS satellites and broadcast correction information. Second, CMM is nomadic, i.e., the network among Stations and Rovers is set up in an ad hoc manner anywhere anytime. Finally, CMM works in Nav2Nav which means there is no need for third party services. However, one limitation of CMM is that navigation systems in Nav2Nav must contain maps with high positional accuracy and resolution.

\section{Future Research}

CMM in its current version merely projects GPS points onto road segments to calculate PRCs. In our future research we will improve CMM performance by taking into account road attributes such as lane width and number of lanes. Interest in GPS prediction models using 3D terrain/city model data has recently increased, e.g., see [4] and [1]. Involving such prediction models in CMM to improve its performance is another topic of future research.

\section{REFERENCES}

[1] D. Roongpiboonsopit, "Navigation Recommender: Real-Time IGNSS QoS Prediction for Navigation Services," Ph.D. dissertation, School of Information Sciences, Univ. Pittsburgh, Pittsburgh, PA, 2011.

[2] W. Chen, M. Yu, Z.-L. Li and Y.-Q. Chen, "Integrated vehicle navigation system for urban applications," in Proc. $7^{\text {th }}$ Int. Conf. Global Navigation Satellite Systems (GNSS), European Space Agency, Graz, Austria, pp. 15-22, April 22-24, 2003.

[3] L. Zhao, W.Y. Ochieng, M.A. Quddus, and R.B. Noland. "An extended Kalman filter algorithm for integrating GPS and low-cost dead reckoning system data for vehicle performance and emissions monitoring," The Journal of Navigation, Royal Institute of Navigation, vol. 56, no. 2, pp.257-275, 2003.

[4] G. Taylor, J. LI, D. Kidner, C. Brunsdon, and M. Ware, "Modelling and prediction of GPS availability with digital photogrammetry and LiDAR," International Journal of Geographical Information Science, vol. 21, no. 1, pp.1-20, Jan. 2007.

[5] E. Kaplan, C. Hegarty, Understanding GPS: principles and applications. 2nd ed. Norwood, MA: Artech House, Inc., 2006, pp. 379-380.

[6] P. A. Zandbergen, D. A. Ignizio, K. E. Lenzer, "Positional Accuracy of TIGER 2000 and 2009 Road Networks," Transactions in GIS, vol. 15, no.4, pp. 495-519, 2011.

[7] NAVTEQ's NAVSTREETS Street Data Reference Manual v.2.0, NAVTEQ, Chicago, Illinois, 2006.
[8] Penn State Institutes of Energy and the Environment. (2009). Pennsylvania Spatial Data Access [Online]. Available: http://www.pasda.psu.edu/

[9] H. A. Karimi, T. Conahan, and D. Roongpiboonsopit, "A Methodology for Predicting Performances of Map-Matching Algorithms," in Proc. $6^{\text {th }}$ Int. Symposium on Web and Wireless Geographical Information Systems (W2GIS 2006),” Hong Kong, pp. 202-213, December 4-5, 2006.

[10] U.S. Coast Guard Navigation Center. (2011, June 6). GPS Almanac Information [Online]. Available: http://www.navcen.uscg.gov/?pageName=gpsAlmanacs

[11] T.H. Witte, A.M. Wilson, "Accuracy of WAAS-enabled GPS for the determination of position and speed over ground," Journal of Biomechanics, vol. 38, pp. 1717-1722, 2005.

[12] Constell, Inc. Constellation Toolbox [Online]. Available: http://www.constell.org/

[13] Refractions Research, Inc. PostGIS [Online]. Available: http://www.postgis.org/

[14] PostgreSQL [Online]. Available: http://www.postgresql.org/

[15] C. Fan, N. Kubo, K. Sasano, T. Tanaka, H. Hasegawa, H. Namie, "Vehicle Positioning by Network-Based RTK-GPS Using Area Correction Parameter (FKP) via TV Wave in Japan," in Proc. of the 2004 National Technical Meeting of The Institute of Navigation, San Diego, CA, January 2004, pp. 475-482.

[16] H. Namie, Naoto Tanaka, Akio Yasuda, "RTK-GPS Positioning in Japan by GPS-Based Control Station via DMCA Mobile Radio Communication System,” in Proc. of the 1999 National Technical Meeting of The Institute of Navigation, San Diego, CA, 1999, pp.495503.

[17] G. Retscher, "Accuracy Performance of Virtual Reference Station (VRS) Networks," Journal of Global Positioning Systems, vol. 1, no. 1, pp. 4047, 2002.

[18] G.R. Hu, V. H.S. Khoo, P. C. Goh, C. L. Law, "Internet-based GPS VRS RTK Positioning with a Multiple Reference Station Network," Journal of Global Positioning Systems, vol. 1, no. 2, pp. 113-120, 2002.

[19] L. Wanninger, "Virtual Reference Stations for Centimeter-Level Kinematic Positioning," in Proc. of ION GPS, 2002, Portland, Oregon, pp. 1400-1407.

[20] Federal Aviation Administration. (2010, August 16). Navigation Services - Wide Area Augmentation System (WAAS) [Online]. Available:

http://www.faa.gov/about/office org/headquarters offices/ato/service u nits/techops/navservices/gnss/waas/

[21] E. Kaplan, C. Hegarty, Understanding GPS: principles and applications. 2nd ed. Norwood, MA: Artech House, Inc., 2006, pp. 432-435.

[22] F. Toran and J. Ventura. The ESA SISNeT: Current Status and Future Plans [Online]. Available: http://www.egnospro.esa.int/Publications/SISNET/ENC_2006_Toran_Ventura_SISNeT_f inal.pdf

[23] H. A. Karimi, B. Zimmerman, D. Nawn, and P. Sutovsky, "Collaborative Navigation Systems for Collision Avoidance", in Proc. $5^{\text {th }}$ Int. Conf. on Collaborative Computing: Networking, Applications and Worksharing, Chicago, Illinois, October 9-12, 2010.

[24] K. Lakakis, P. Savvaidis, I. M. Ifadis, and I. D. Doukas, "Quality of Map-Matching Procedures Based on DGPS and Stand-Alone GPS Positioning in an Urban Area," in Fèdèration Internationale des Gèometres Working Week 2004, Athens, Greece, May 22-27, 2004.

[25] H. A. Karimi, D. Roongpiboonsopit, and P. Kasemsuppakorn, "Uncertainty in Personal Navigation Services," Journal of Navigation vol. 64, pp. 341-356, 2011. 